\title{
Estomatitis protésica: puesta al día
}

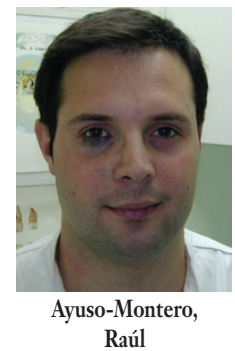

Denture stomatilis: update

\section{Alusso-Montero, Rä́l* Torrent-Collado, Jose $\mathrm{e}^{* \star}$ López-López, José $e^{* * *}$}

* Odontólogo. Máster en Oclusión y Rehabilitación Oral. Universidad de Barcelona.

** Médico-Estomatólogo. Profesor asociado de Prótesis. Universidad de Barcelona.

*** Médico-Estomatólogo. Profesor Titular de Medicina Bucal. Facultad de Odontología de la Universidad de Barcelona.
Resumen: La estomatitis protésica es una inflamación crónica de la mucosa oral debida al contacto con una prótesis extraible. Es un proceso difícil de controlar en los pacientes portadores de este tipo de prótesis. La patología involucra diferentes factores predisponentes, y el más importante de ellos es la presencia de Candida. En el presente artículo se describe el manejo del paciente y las dificultades de los tratamientos actuales para eliminar de las prótesis y de la mucosa oral los residuos fúngicos.

Se concluye que los hábitos de higiene oral y personal en pacientes portadores de prótesis extraible son los métodos principales para evitar la aparición de la patología. Una vez instaurada, podemos controlar al paciente con medidas higiénicas y agentes antifúngicos, pero debemos ser conscientes de las limitaciones terapéuticas ante los depósitos de Candida de las prótesis. En algunos casos puede estar indicada la sustitución de la prótesis por una nueva.

Palabras clave: Estomatitis protésica, Candidiasis protésica, Candidiasis subplaca.

Abstract: Denture stomatitis is a chronic inflammation of the denture-bearing mucosa This pathology is difficult to control by denture wearing patients. The disease envolves different predisposing factors, the most important of them being the presence of Candida.

This article describes the management of these patients and the difficulties of the present treatments in eliminating the fungal remnants from the prosthesis and the oral mucosa.

It is concluded that the oral and personal hygiene habits are the most important methods to prevent this pathology in denture wearing patients. If the pathology is already established, we can control these patients by means of hygiene measures and antifungal agents, but we have to be aware of the therapeutic limits in presence of Candida deposits on the prostheses. In some cases, replacement of the prosthesis may be indicated.

Key words: Denture stomatitis, Candida stomatitis.

\begin{tabular}{ccc}
\hline Fecha recepción & Fecha última revisión & Fecha aceptación \\
$29-12-2003$ & $27-07-2004$ & $4-11-2004$ \\
\hline
\end{tabular}

Raúl Ayuso Montero

Po Fabra i Puig 472, 30 3a

08042 Barcelona

E-mail: ayusoden@hotmail.com 


\section{Introducción}

La estomatitis por prótesis es una inflamación crónica de la mucosa oral que está en contacto con prótesis

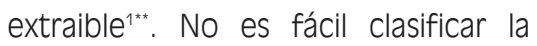
estomatitis protésica (fig.1) (también denominada estomatitis sub-placa) dentro del abanico de la patología bucal. El hecho de estar relacionada con múltiples factores etiológicos hace que en la literatura se clasifique dentro del resto de estomatitis, pero su relación directa con las micosis candidiásicas hace que normalmente la encontremos como una variedad de candidiasis.

Afecta al $14,3 \%$ de la población mayor de 60 años según Jainkittivong $^{2}$, y en nuestro medio al $6,5 \%$ de la población mayor de 30 años $^{3}$. Si hablamos sólo de los portadores de prótesis, afecta al $67 \%$ según Pires ${ }^{4 *}$, y al $50,31 \%$ en nuestro medio ${ }^{5^{*}}$.

Lo cierto es que hay varios factores predisponentes, como son la falta de higiene oral, la edad (que conlleva normalmente el consumo elevado de medicamentos que inducen un flujo salivar reducido), infecciones por Candida, diabetes, déficits inmunológi$\cos ^{4^{*}, 5^{*}, 6}$, consumo de tabaco y tener colocada la prótesis por la noche ${ }^{7 *}$. La patología siempre se presenta en portadores de prótesis extraibles (que pueden provocar traumatismos de repetición, reacciones alérgicas y pueden suponer un nicho para los microorganismos y residuos alimentarios cuando sus superficies son rugosas).

De todos los factores, el más directamente relacionado con la estomatitis protésica es la presencia de especies de Candida, que está presente en la cavidad oral del $25-50 \%$ de sujetos

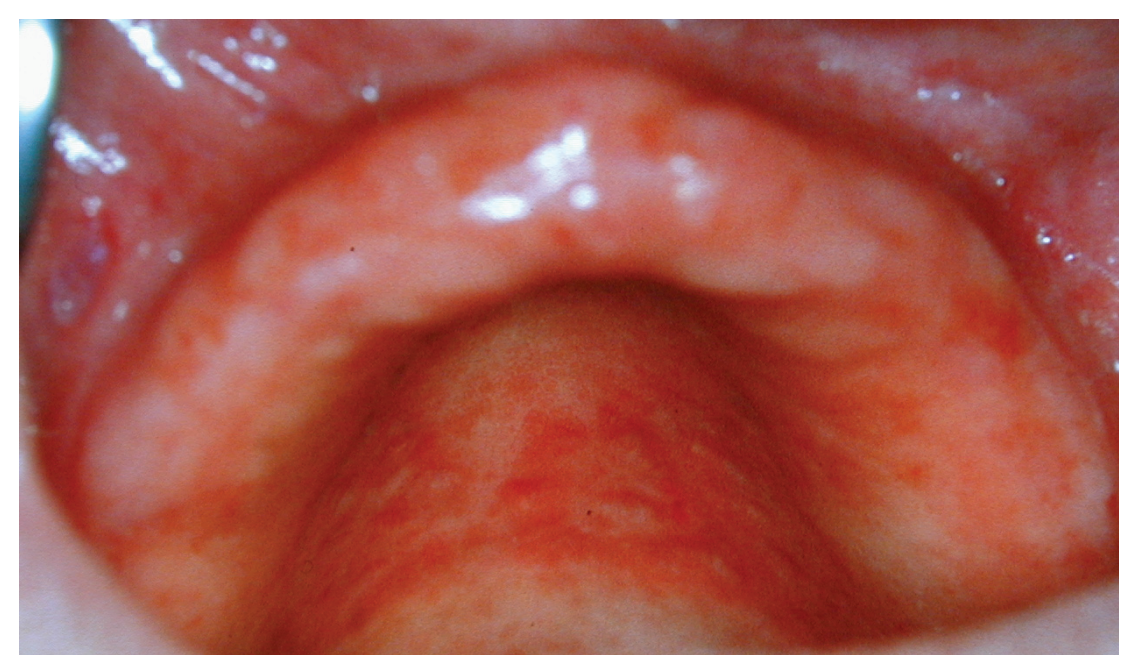

Figura 1. Inflamación de la mucosa palatina.

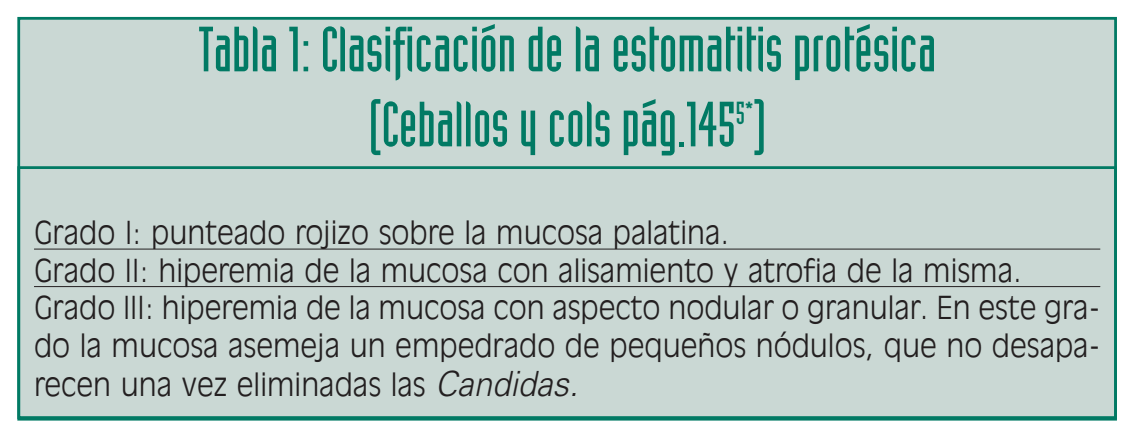

sanos según Pires ${ }^{4 *}$ y en el 30-70\% según Darwazeh ${ }^{8}$. Ésta proporción aumenta en pacientes portadores de prótesis extraibles, desde el $34,27 \% 5^{*}$ hasta el $60-100 \%^{4^{*}}$.

En base a estos aspectos Darwazeh cita que el $71,4 \%$ de los pacientes diagnosticados de estomatitis subplaca protésica presentan colonización por Candida ${ }^{8}$.

Desde el punto de vista clínico, tal y como recogen en su libro Ceballos y cols se acepta la clasificación propuesta por Newton ${ }^{5 *}$, que dividió la estomatitis sub-placa en tres fases: grado I, grado II y grado III (tabla 1).

\section{Faclores predisponentes}

Resumiremos a continuación los datos más relevantes referentes a los factores predisponentes:

\section{Higiene oral deficiente}

La estomatitis protésica aparece más frecuentemente en portadores de prótesis con mala higiene oral. La presencia de placa bacteriana favorece la colonización por parte de Candidas tanto en la superficie de las prótesis como en la mucosa. Kulak estudió la relación entre la estomatitis protésica y la higiene ora ${ }^{9^{*}}$; monitorizó en 70 pacientes por- 
tadores de prótesis completas la presencia de estomatitis, la presencia de hongos, el método y la frecuencia de limpieza de la prótesis y el estado de la misma. Concluyó que no había relación directa entre el método de limpieza y frecuencia de lavado de la prótesis con la aparición de la enfermedad; sin embargo, sí había relación significativa entre la presencia de suciedad, la colonización de prótesis y mucosa por levaduras y la presencia de estomatitis protésica. El autor deduce, pues, que no importa el método ni frecuencia de limpieza mientras la prótesis se mantenga limpia.

Al manipular la prótesis para colocársela, los pacientes pueden infectarse las superficies dactilares por Candida, y crear así un círculo de reinfección sucesiva entre los dedos y la cavidad oral y viceverså. Por eso es importante insistir en las medidas de higiene personal entre las que se incluye un correcto lavado de las manos.

La implantación de programas preventivos de salud oral en los pacientes de edad avanzada nos permitiría prevenir la enfermedad. Las medidas de estos programas incluyen el adiestramiento de los cuidadores y responsables de estos pacientes cuando son dependientes de terceras personas ${ }^{10^{* * *}}$.

\section{Factores dependientes del huésped}

La Candida tiene mayor capacidad de infección si el terreno le es favorable. Se sabe que los pacientes de edad avanzada presentan mejores condiciones para desarrollar la patología. Su flujo salival es reducido, con lo cual carecen de lisozimas, lactoferrina ${ }^{11} y$ las citoquinas salivare ${ }^{4 * 12}$ que inhiben y controlan el crecimiento de las Candidas, y normalmente no tienen las

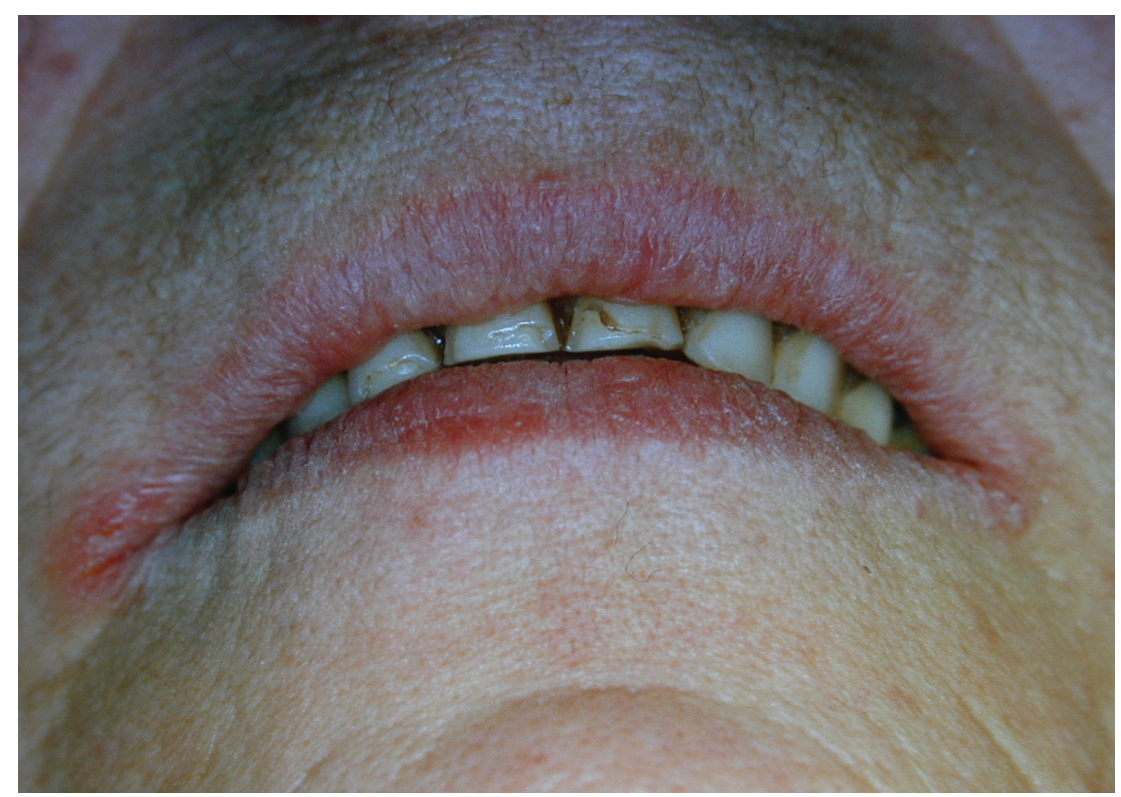

Figura 2 a. Se puede observar la prótesis deteriorada colocada en boca y rágades en las comisuras labiales.

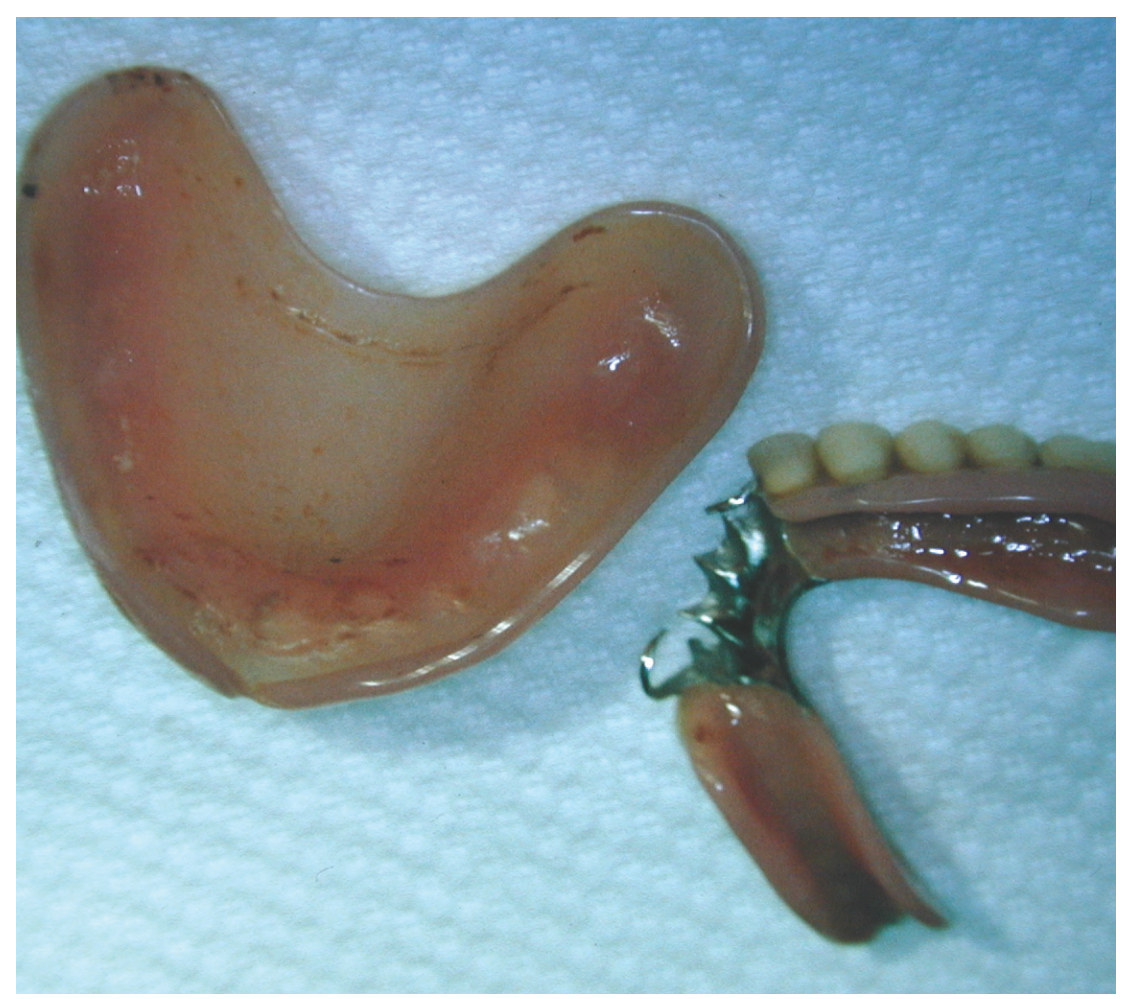

Figura 2b. Obsérvese la acumulación de residuos en la superficie de la prótesis que contacta con la mucosa oral 
mejores condiciones higiénicas. Además, la formación de una película salival sobre todas las superficies es un método de protección para la cavidad oral. La unión de inhibidores de las proteasas al polimetilmetacrilato de las prótesis varía entre individuos, y esto explica la mayor susceptibilidad de algunos sujetos a la colonización por parte de los hongos ${ }^{13}$. Los sujetos con problemas de inmunocompetencia y con enfermedades sistémicas asociadas, tales como la diabetes tienen problemas similares. La inmunidad celular mediada por las células $T$ helper (CD4+) activa las citoquinas salivares Th1 y Th2, que se consideran las responsables de la resistencia a la infección por Candida ${ }^{12}$.

El hecho de no quitarse la prótesis por las noches y de fumar son los dos factores que provocan mayor inflamación y una clínica más exagerada según el trabajo de Barbeau ${ }^{7 *}$. Su estudio consistió en el análisis estadístico de relación entre los factores predisponentes y la presencia de inflamación y su extensión, sin tener en cuenta la clasificación de Newton.

\section{Factores dependientes de la prótesis}

El simple hecho de portar la prótesis ya es un factor predisponente para la patología. Se crea un ambiente cerrado, más anaerobio, entre la prótesis colocada en la boca y la mucosa, con lo cual se favorece el crecimiento de las Candidas, pudiendo pasar las mismas de ser un hongo comensal en la mucosa a ser un parásito que infecte la mucosa ${ }^{1 * *}$.

Las prótesis extraibles generalmente están formadas en su totalidad o en buena parte por resina de polimetilmetacrilato. Sobre dicho sustrato la
Candida es capaz de generar una matriz extracelular diferente a la que generan sobre otra superficie ${ }^{14 *}$, esta forma de crecimiento se llama biofilm. Dicho biofilm contiene menos proteínas e hidratos de carbono y más glucosa y galactosa que si la Candida creciese en condiciones normales. Estas diferencias explican que el biofilm presente mayor resistencia a los tratamientos antifúngicos, y productos con amfotericina B, nistatina, clorhexidina y fluconazol no han sido capaces de eliminar la Candida en dichas condiciones ${ }^{15,16}$. Si además la superficie de la resina es rugosa y tiene una elevada porosidad, se favorece la acumulación de residuos (figs. 2 a y 2 b) y la aparición de la enfermedad ${ }^{4 *}$. Las prótesis rebasadas con materiales blandos presentan el mismo problema, si bien en mayor grado por la facilidad de deterioro de dichos materiales ${ }^{17}$. La utilización de algunos agentes químicos para su limpieza a veces favorecen el deterioro del material de rebase y no previenen el crecimiento de Candida.

Monsenego presenta un estudio sobre 15 pacientes en el que la aplicación de un barniz fotopolimerizable (Permalink $\otimes$ ) sobre la superficie de la resina reduce el número de bacterias capaces de adherirse a las prótesis ${ }^{18^{*}}$. Barnizó un área de la prótesis en cada paciente y tomó una muestra a los 15 días de la parte barnizada y la no barnizada, así como de la mucosa correspondiente a cada una de las partes, y observó que disminuía de forma significativa la cantidad de colonias en los dos sustratos.

El traumatismo por presión que pueden ejercer algunas prótesis sobre la mucosa hace que ésta sea más susceptible a la aparición de estomati- tes $^{5^{*}}$, y este factor se describe como etiológico en algunos casos, al igual que la irritación por alergia al monómero del acrílico de resina $a^{3,4^{*}}$.

\section{Prevención}

En los pacientes de edad avanzada que son generalmente los portadores de prótesis, es conveniente implantar un programa preventivo adaptado a las necesidades de cada uno $0^{10^{* * *}}$. En dicho programa preventivo se incluyen las siguientes medidas: profilaxis y eliminación de los residuos bacterianos en los dientes remanentes y en las prótesis en la consulta dental en intervalos máximos de seis meses (dependiendo del paciente); recambio de los cepillos dentales y los cepillos de limpieza de las prótesis; instrucciones de higiene oral y de la prótesis a pacientes y cuidadores, incluyendo el dejarla seca ${ }^{10^{*+}} y$ no colocársela de noche ${ }^{7 *}$ y tener un correcto lavado de manos ${ }^{8}$. Según el estudio de Barbeu, el tabaquismo es uno de los factores de riesgo que se relaciona con una clínica más agresiva $^{7^{*}}$, por lo que deberíamos aconsejar a éstos pacientes dejar de fumar.

Se debe recomendar al paciente dejar la prótesis seca al aire durante ocho horas por las noches consigue reducir considerablemente la cantidad de Candidas de la prótesis, podemos además aconsejarle que la coloque periódicamente en clorhexidina al $0,2 \%{ }^{1 * *}$.

\section{Tratamiento}

Consiste en enseñar al paciente las medidas higiénicas individuales y de la prótesis $^{4,5^{*}}$. Se le debe indicar dormir 


\begin{tabular}{|c|c|}
\hline Tabl & 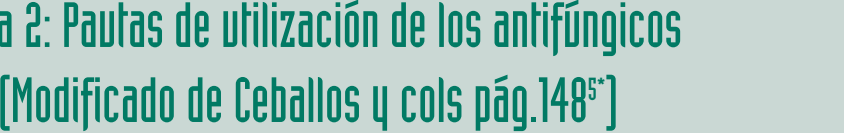 \\
\hline Nistatina: & $\begin{array}{l}\text { Suspensión oral ( } 100.000 \mathrm{U} / \mathrm{ml}) \quad 5-15 \mathrm{ml} / \text { día, enjuagues de } \\
1^{\prime} \text { y deglutir, cada seis horas } \\
\text { Comprimidos ( } 200.000 \mathrm{U} / \mathrm{comp}) \quad 1-3 \mathrm{comp} / \text { día disueltos en } \\
\text { la boca }\end{array}$ \\
\hline Miconazol: & $\begin{array}{l}\text { Comprimidos } 500 \mathrm{mg} 2 \mathrm{comp} / \text { día disueltos en la boca } \\
\text { Gel oral } 2 \% \quad 1-2 \text { aplicaciones/día }\end{array}$ \\
\hline Clotrimazol: & Comprimidos $10 \mathrm{mg}$ 4-5 comp/día disueltos en la boca \\
\hline Amfotericina B: & Vía endovenosa $5 \mathrm{mg} / \mathrm{kg} / \mathrm{día}$ \\
\hline Ketoconazol: & Comprimidos $200 \mathrm{mg}$ 1comp/día \\
\hline Fluconazol: & $\begin{array}{l}\text { Cápsulas } 50 \mathrm{mg} \text { 1cáp/día } \\
150 \mathrm{mg} \text { dosis única }\end{array}$ \\
\hline Itraconazol: & Tabletas 100 mg 2/día \\
\hline
\end{tabular}

sin la misma, colocando la prótesis en una solución de clorhexidina a concentración entre el 0,2 y el $2 \%$ o de hipoclorito de sodio entre el 0,5 y el $2 \%^{1 * *}$. Ésta última opción no es compatible con las prótesis metálicas ${ }^{19}$. Los agentes antifúngicos pueden ser tópicos o sistémicos, y está demostrada la misma efectividad terapéutica para el mismo agente por ambas vías de administración en pacientes inmunocompetentes $^{20}$.

Los antifúngicos pueden ser agentes poliénicos (nistatina y amfotericina B), imidazoles (clotrimazol, miconazol y ketoconazol) o triazoles (fluconazol e itraconazol) $)^{5 * 11}$, aunque conocemos la resistencia del biofilm a dichos tratamientos ${ }^{14 * 15,16}$.
Indicaremos tratamiento antifúngico cuando el paciente refiera una clínica de dolor o sensación de ardor, o bien exista riesgo de infección faríngea o sistémica ${ }^{\text {t**}}$ y se tiene que alargar cuatro semanas. Las pautas de tratamiento de los diferentes antifúngicos se presentan en la tabla 2 .

Existe una planta conocida como Punica granatum que ha demostrado efectos antifúngicos similares al miconazo ${ }^{21}$ en pacientes con estomatitis protésica. En dos grupos de 30 pacientes cada uno, Vasconcelos y cols trataron a uno de ellos con miconazol y a otro con Punica, obteniendo resultados semejantes, si bien la obtención de la misma y la preparación del unguento se supone más dificultosa.
Hay casos en que existe la necesidad de sustituir la prótesis. El simple hecho de reemplazarla por una nueva ha sido suficiente para eliminar el proceso en algunos pacientes ${ }^{4 *}$. Pires $y$ cols demostraron que en seis meses el porcentaje de pacientes con estomatitis protésica bajó del $50,6 \%$ al 18,2\% en un estudio sobre 77 pacientes en los que sustituyó la prótesis.

\section{Conclusiones}

1. Se acepta la presencia de Candida como el factor predisponente más influyente en la estomatitis protésica.

2. La higiene oral y personal, así como la limpieza de las prótesis, permiten la eliminación de residuos fúngicos de la cavidad oral y de superficies corporales como las manos, que intervienen en la manipulación de las prótesis. Siendo así, la higiene se presenta como el principal método preventivo y terapéutico.

3. Deberíamos utilizar agentes antifúngicos cuando los cambios en los hábitos higiénicos no son capaces por sí solos de resolver el cuadro clínico o cuando la agresividad de la clínica así lo justifique.

4. En algunos casos, está indicado el cambio de las prótesis, dada la dificultad de eliminar los residuos de la resina de las prótesis.

\section{Bibliografia recomendada}

Para profundizar en la lectura de este tema, el/los autor/es considera/an interesantes los artículos que aparecen señalados del siguiente modo: *de interés ** de especial interés.

1.** Budtz-Jorgensen E. Candida -associated denture stomatitis and angular cheilitis. Samaranayake LP, MacFarlane T. En: Samaranayake
LP, MacFarlane TW. Oral candidosis. London: Wright 1990:cap 9.

En este capítulo, el autor resume los métodos de diagnóstico, factores predisponentes, recomendaciones preventivas y tratamiento.

2. Jainkittivong A, Aneksuk V, Langlais RP. Oral 
mucosal conditions in elderly dental patients. Oral Dis 2002;8:218-23.

3. García-Pola Vallejo MJ, Martínez Díaz-Canel AI, García Martín JM, González García M. Risk factors for oral soft tissue lesions in adult Spanish population. Community Dent Oral Epidemiol 2002;30:277-85

4.* Pires FR, Santos EB, Bonan PR, De Almeida OP, Lopes MA. Denture stomatitis and salivary Candida in brazilian edentulous patients. J Oral Rehabil 2002;29:1115-9. En este estudio, el autor analiza el porcentaje de pacientes con estomatitis protésica de un grupo de 77 pacientes. El resultado es del 50,6\%. A todos ellos les sustituye la prótesis por una nueva y vuelve a hacer el recuento seis meses después, obteniendo un $18,2 \%$.

5.* Ceballos Salobreña A, Delgado Azareño W, Gándara Vila P. Micosis bucales. En: Ceballos Salobreña A, Bullón Fernández P, Gándara Rey JM, Chimenos Küstner E, Blanco Carrión A, Martínez-Sahuquillo Márquez A, García García A. Medicina Bucal Práctica. Santiago de Compostela: Danú, 2000:144-5.

En el capítulo se clasifican las micosis bucales y se incluye a la estomatitis protésica en micosis asociada a otras lesiones. Además, muestra la clasificación de Newton y los tratamientos y posologías utilizadas para las micosis orales.

6. Escovich L, Ceballos A. Candida albicans y precáncer oral. En: Bascones A, Seoane JM, Aguado A, Suárez-Quintanilla JMª . Cáncer y precáncer oral. Bases clínico-quirúrgicas y moleculares. Madrid: Avances médico-dentales, 2003:76

7.* Barbeau J, Seguin J, Goulet JP, de Konink L, Avon SL, Lalond B, Rompre P, Deslauriers N. Reassessing the presence of Candida albicans denture related stomatitis. Oral Surg Oral Med Oral Pathol Oral Radiol Endod 2003; 95:51-9.

En este estudio el autor analiza la relación de los factores de riesgo con la aparición de estomatitis protésica y su grado de estomatitis protésica.
Concluye que sólo el hecho de fumar y de llevar la prótesis de noche aumentan el grado de inflamación de la mucosa.

8. Darwazeh AM, Al-Refai S, Al-Mojaiwel S. Isolation of Candida species from the oral cavity and fingertips of complete denture wearers. J Prosthet Dent 2001;86:420-3.

9.* Kulak-Ozkan Y, Kazazoglu E, Arikan A. Oral hygiene habits, denture cleanliness, presence of yeasts and stomatitis in elderly people. J Oral Rehabil 2002;29:300-4

En su estudio, el autor analiza la influencia sobre la estomatitis protésica de la presencia de levaduras en la dentadura y la frecuencia y método de limpieza de la prótesis. Concluye que la patología no depende de la frecuencia ni método de limpieza, pero sí de el hecho de que la dentadura contenga o no levaduras.

10.** Butz-Jorgensen E, Mojon P, Rentsch A, Deslauriers N. Effects of an oral health program on the ocurrence of oral candidosis in a longterm care facility. Community Dent Oral Epidemiol 2000;28:141-9.

En su estudio, el autor demuestra que establecer un programa de salud oral con medidas didácticas e higiénicas de prótesis y cavidad oral, tanto para pacientes como para cuidadores, se reduce la incidencia de colonizaciones por Candida de un $48,7 \%$ a un $23,4 \%$

11. Samaranayake YH, Saramanayake LP. Experimental oral candidiasis in animal models. Clin Microbiol Rev 2001;14:398-429.

12. Leigh JE, Steele C, Wormley F, Fidel PL Jr. Salivary cytokine profiles in the inmunocompetent individual with Candida associated denture stomatitis. Oral Microbiol Inmunol 2002; $17: 311-4$

13. Göcke R, Gerath F, von Schwanewede H. Quantitative determination of salivary components in the pellicle on PMMA denture base material. Clin Oral Investig 2002;6:227-35.

14. *Baillie GS, Douglas LJ. Matrix polymers of Candida biofilms and their possible role in biofilm resistance to antifungal agents. $\mathrm{J}$ Antimicrob Chemother 2000;46:397-3.

Analiza el comportamiento de dos películas de Candida, una en forma de biofilm y otra en forma normal o planctónica ante un tratamiento con anfotericina B. Concluye que la forma de biofilm es más resistente al tratamiento.

15. Chandra J, Mukherjee PK, Leidich SD y cols. Antifungal resistance of candidal biofilms formed on denture acrylic in vitro. J Dent Res 2001;80:903-8

16. Mukherjee PK, Chandra J, Kuhn DM, Ghannoum MA. Mechanism of Fluconazol resistance in Candida albicans biofilms: phase-specific role of efflux pumps and membrane sterols. Infection and Immunity 2003;71:4333-40.

17. Nikawa H, Jin C, Makihira S, Egusa H, Hamada T, Lumagai H. Biofilm formation of Candida albicans on the surfaces of deteriorated soft denture lining materials caused by denture cleansers in vitro. J Oral Rehabil 2003; 30:243-50.

18.* Monsenego P. Presence of microorganisms on the fitting denture complete surface: study in vivo. J Oral Rehabil 2000;27:708-13. Explica la importancia del depósito de microorganismos sobre las superficies de las prótesis y demuestra que con una superficie más lisa (barnizada) se acumulan menos colonias de microorganismos.

19. Porta J. Asepsia en el laboratorio. En: Porta J. Asepsia en odontología. Barcelona: COEC 1994:44.

20. Cross LJ, Bagg J, Oliver D, Warnock D. Serum itraconazole concentrations and clinical responses in Candida-associated denture stomatitis patients treated with itraconazole solution and itraconazole capsules. J Antimicrob Chemother 2000;45:95-9.

21. Vasconcelos LC, Sampaio MC, Sampaio FC, Higinio JS. Use of Punica granatum as an antifungal agent against candidosis associated with denture stomatitis. Mycoses 2003;45:192-6. 